\title{
Habitual dietary patterns of patients on hemodialysis indicate nutritional risk
}

\begin{abstract}
Objective: This study aimed to (i) determine habitual dietary patterns of Malaysian patients on hemodialysis (HD) and (ii) examine their association with nutritional status. Methods: An à posteriori approach examined 3-day dietary recalls of 382 multiethnic Malaysian patients on HD, leading to short-listing of 31 food groups. Dietary patterns were derived through principal component analysis. Sociodemographic and lifestyle characteristics together with nutritional parameters were examined for associations with specific dietary patterns. Results: Four dietary patterns emerged, namely, "Home Food," "Eating Out (EO)-Rice," "EO-Sugar sweetened beverages," and "EO-Noodle." Younger patients, male gender, Malay, and patients with working status were more likely to follow "EO-Rice" and "EO-Sugar sweetened beverages" patterns, while Chinese patients were more likely to consume "EO-Noodle" pattern (all P values $<.05$ ). The EO frequency was directly associated with "EO-Rice" $(\mathrm{P}=.030)$, "EO-Sugar sweetened beverages" $(\mathrm{P}=.040)$, and "EO-Noodle" $(\mathrm{P}=.001)$ patterns. The highest tertile of the "Home Food" pattern related to higher handgrip strength $(\mathrm{T} 3=21.3 \pm 0.74$ vs. $18.0 \pm 0.73 \mathrm{~kg}, \mathrm{P}=.006)$, higher serum albumin $(\mathrm{T} 3=3.99 \pm 0.04$ vs. $\mathrm{T} 1=3.84 \pm 0.04 \mathrm{~g} / \mathrm{dL}, \mathrm{P}=.027)$, and lower MalnutritionInflammation Score $(\mathrm{T} 3=4.9 \pm 0.36$ vs. $\mathrm{T} 1=6.4 \pm 0.34, \mathrm{P}=.010)$, along with lower Diet Monotony Index (T3 = 29.0 \pm 1.1 vs. T1 = 33.0 $\pm 1.0, \mathrm{P}=.030)$. while "EO-Rice" and "EO-Sugar sweetened beverage" patterns were associated only with higher energy intake (all P values $<.001$ ). Conclusions: These results indicated that a home-based diet inclusive of healthy food choices was associated with better nutritional status in this HD population.
\end{abstract}

